\title{
Fístula aorto pulmonar: endocarditis izquierda en paciente VIH y ADVP. Revisión de la literatura
}

\author{
B. OBÓN AZUARA, B. ZALBA ETAYO, I. GUTIÉRREZ CÍA, B. VILLANUEVA ANADÓN
}

Servicio de Medicina Intensiva. Hospital Clínico Universitario. Zaragoza

\begin{abstract}
AORTO PULMONARY FISTULA: LEFT-SIDED INFECTIVE ENDOCARDITIS IN HIV AND INTRAVENOUS DRUGS ABUSER PATIENT. REVIEW OF LITERATURE
\end{abstract}

\section{RESUMEN}

La endocarditis infecciosa (EI) es una de las complicaciones más severas en la población adicta a drogas por vía parenteral (ADVP). La infección por el VIH incrementa el riesgo de aparición en los pacientes que además son ADVP. La EI en ambas poblaciones posee una especial tendencia a infectar las válvulas del hemicardio derecho, siendo infrecuente la afectación aórtica.

Se expone el caso un paciente VIH y ADVP, que ingresa por síndrome febril, con Rx de tórax inicial normal y hemocultivos negativos. CD4 $90 \mathrm{~mm}^{3}$. Imposible realizar ecocardiograma transesofágico (ETE), revelando el transtorácico (ETT) una insuficiencia aórtica moderada con función sistólica conservada. A pesar de antibioterpia de amplio espectro, antifúngico y tratamiento antirretroviral (TAR) presentó SDRA por lo que es intubado. Se realizó ETT apreciando una gran desestructuración aórtica y una fístula aorto-pulmonar secundaria a una EI izquierda. Posteriormente solo un hemocultivo fue positivo para $S$. aureus. Fue desestimado el tratamiento quirúrgico. El paciente falleció tras 3 semanas de evolución.

PALABRAS CLAVE: Endocarditis infecciosa izquierda. Fístula aortopulmonar. Stafilococo aureus. VIH y ADVP.

\begin{abstract}
Infective endocarditis (IE) is the most severe complication in intravenous drug abusers (IVDAs). HIV infection increases the risk of IE in IVDAs too. IE in both population are special tendency to infect the rigthsided heart, but unusual infective aortic valve. We report a case of HIV and IVDA patient admitted in hospital due to fever syndrome, with X-ray test normal and the first blood cultures negatives. CD4 count cell 90 $\mathrm{mm}^{3}$. It was impossible doing a transesophageal echocardiography (TEE) and transtoracic echocardiogramma (TTE) only showed a moderate aortic insufficiency with conserved systolic function. Despite using antibiotics, antifungals and highly active antirretroviral therapy, he developed ARDS, and mechanical ventilation should be performed. At that moment, TEE showed an aorto pulmonary fistula due to left-sided IE. Further cultures was undergone and only one blood culture was positive to Staphylococcus aureus. Cardiac surgery was not indicated. The patient died 3 weeks later.
\end{abstract}

KEY WORDS: Left-sided infective endocarditis. Aorto pulmonary fistula. Staphylococcus aureus. HIV and intravenous drugs habit.

Obón Azuara B, Zalba Etayo B, Gutiérrez Cía I, Villanueva Anadón B. Fístula aorto pulmonar: endocarditis izquierda en paciente VIH y ADVP. Revisión de la literatura. An Med Interna (Madrid) 2007; 24: 547-550.

\section{INTRODUCCIÓN}

La endocarditis infecciosa (EI) en personas adictas a drogas por vía parenteral (ADVP) es una de las complicaciones más severas en este grupo de población $(1,2)$. Aunque el VIH poor sí mismo no es un factor de riesgo para el desarrollo de EI, la propia infección por el VIH sí que incrementa el riesgo de EI en los pacientes que además son ADVP (2-4). A continuación exponemos un caso de EI en un paciente VIH y ADVP.

\section{CASO APORTADO}

Paciente de 38 años de edad, VIH positivo desde hace 20 años en tratamiento antirretroviral de forma discontinua, usuario ocasional de drogas (metadona oral y heroína intravenosa), y hepatitis por virus B y C. La semana previa a su ingreso había consumido heroína, siendo ingresado en el hospital por síndrome febril.

Cuenta con diversas exploraciones complementarias realizadas en el hospital antes de ser ingresado en UCI, entre ellas dos ecocardiografías transtorácicas efectuadas con una semana de diferencia, en las que existe una insuficiencia aórtica moderada con alteración

Trabajo aceptado: 20 de junio de 2007 
estructural de dicha válvula, y una leve insuficiencia tricuspídea, sin existir alteraciones en la contractilidad global. Resulta imposible realizar un ecocardiograma transesofágico (ETE) ante la reiterada negativa del paciente. Además se realiza un estudio de la inmunidad con cifras de CD4 de $90 \mathrm{~mm}^{3}$; coeficiente CD4/CD8 1,15\%. En los hemocultivos existe un crecimiento de Stafilococo aureus. La Rx de torax inicialmente es compatible con la normalidad. Desde el primer instante se instaura tratamiento con antibioterapia (ceftriaxona, cloxacilina, sulfametoxazol), a pesar de ello presenta una evolución poco favorable por lo que es ingresado en la Unidad de Cuidados Intensivos debido a una insuficiencia respiratoria global.

A su ingreso en UCI, además de fiebre de $39^{\circ} \mathrm{C}$, destaca la existencia de un soplo sistólico mitral y diastólico aórtico, que no existía previamente. En la Rx de tórax aparece un infiltrado intersticial bilateral con derrame pleural bilateral. En los diferentes cultivos obtenidos en UCI (telescopado, broncoaspirado y punta de catéter central) se encuentra candida albicans en cantidades superiores a 100 unidades formadoras de colonias, con hemocultivos repetidamente negativos, si bien en el último hemocultivo obtenido se halló Stafilococo aureus. Además de antibioterapia de amplio espectro, se añade tratamiento antiviral y antifúngico. Se practica nuevamente una ecocardiografía transtorácica la que se aprecia una válvula aórtica de morfología tricúspide asimétrica, con engrosamiento y prolapso del velo coronario derecho que condiciona una insuficiencia aórtica importante (Fig. 1), con un ventrículo izquierdo no dilatado y una buena contractilidad, con una fracción de eyección del 53\%. Ante la importancia de la insuficiencia respiratoria que aparece durante la evolución es preciso intubarlo y conectarlo a ventilación mecánica. Ante la gravedad de la situación la familia autoriza la realización de un ETE en el que se muestra una endocarditis aórtica con prolapso de la cúspide anterior que produce una regurgitación significativa. Existe además un absceso en el seno de valsalva anterior con un trayecto fistuloso que origina un shunt izquierda-derecha con el tronco de la pulmonar (Fig. 2). Debido a la desestructuración valvular y al shunt aorto-pulmonar, se desestima tratamiento quirúrgico por parte de Cirugía Cardíaca. Finalmente el paciente presenta en su evolución una situación de shock refractario por lo que fallece.

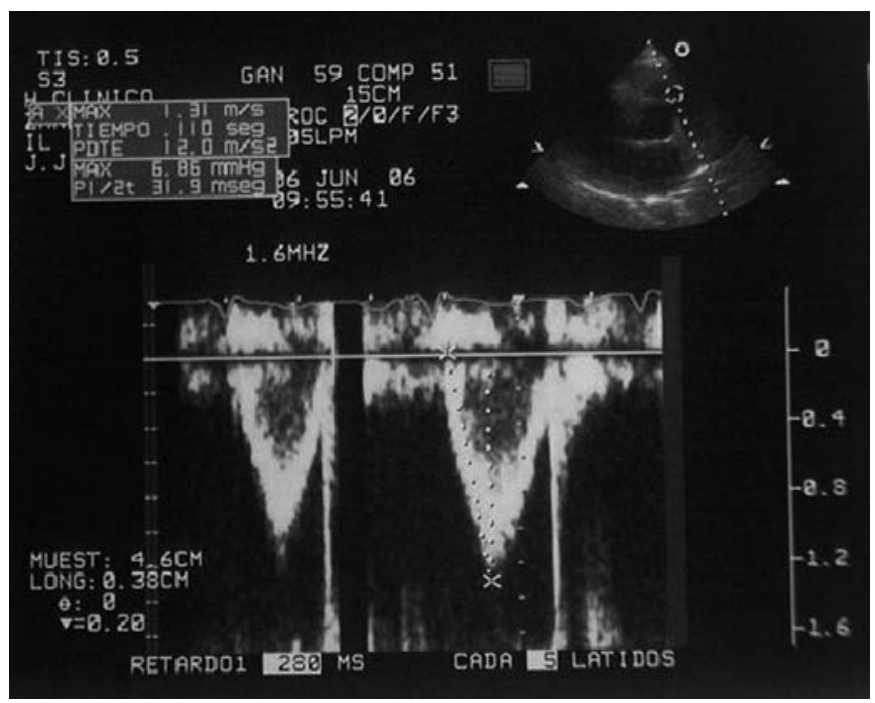

Fig. 1. Ecocardiograma transtorácico: válvula aórtica tricúspide asimétrica, con engrosamiento y prolapso del velo coronario derecho condicionando una insuficiencia aórtica.

\section{DISCUSIÓN}

La endocarditis infecciosa (EI) en personas adictas a drogas por vía parenteral (ADVP) es una de las complicaciones más severas en esta población $(1,2)$. Su incidencia en la pobla-

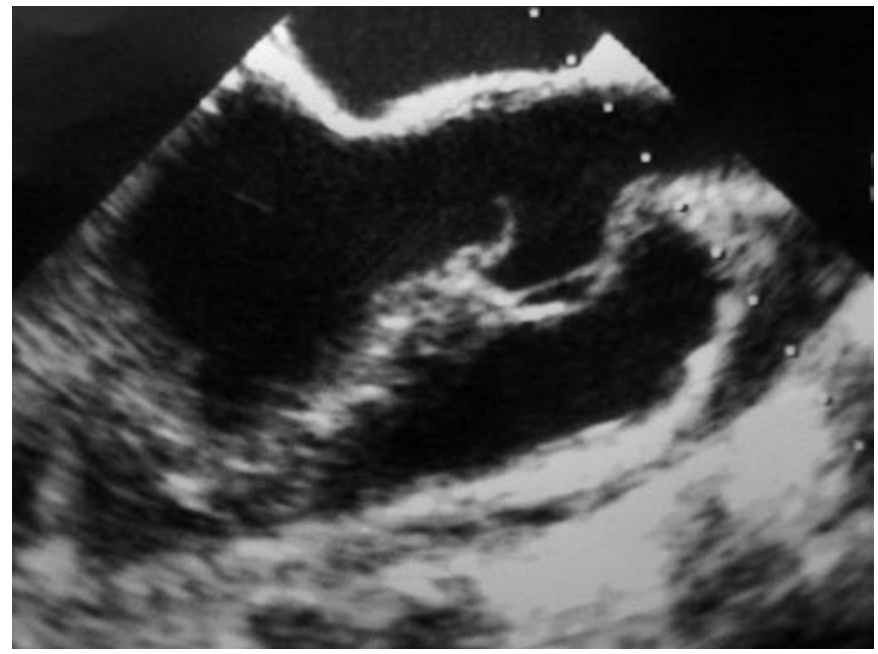

Fig. 2. Ecocardiograma transesofágico: endocarditis aórtica con prolapso de la cúspide anterior que produce una regurgitación. Absceso en el seno de Valsalva anterior con un trayecto fistuloso que origina un shunt izquierda-derecha con el tronco de la pulmonar.

ción ADVP se sitúa entre el 2 al 5\% por año, siendo responsable del 5 al $10 \%$ de la tasa de mortalidad generada por la endocarditis (2-4). Aunque el VIH por sí mismo no es un factor de riesgo para el desarrollo de EI, -siendo ésta más frecuente únicamente en aquellas personas con severa inmunosupresión (cifras de CD4 < a 200) o con criterios de SIDA(5-12), la infección por el VIH sí que incrementa el riesgo de EI en los pacientes que además son ADVP, y así en la actualidad la prevalencia de la EI en los pacientes con infección VIH y ADVP varia del 30 al $90 \%(2,4)$.

La EI en ambas poblaciones posee una especial tendencia a infectar las válvulas del hemicardio derecho. La distribución típica de la infección valvular es tricúspide (90-78\%), siendo menor la frecuencia de la afectación mitral (32-24\%) y aórtica (8-15\%) y afectación simultánea de válvulas derechas e izquierdas $(5 \%)$, todas ellas con porcentajes variables dependiendo de las series consultadas (2-4,6,13-17).

En el $75-93 \%$ de los usuarios DVP las válvulas son sanas antes de la infección. El riesgo de EI en estos individuos es siete veces mayor que en el resto de los individuos inmunocompetentes portadores de una cardiopatía reumática o prótesis valvular. La clínica depende de la válvula afectada y en menor grado del agente causal. A diferencia de la EI del resto de la población adulta, el Stafilococo aureus es el causante en más de la mitad de las infecciones en este tipo de pacientes, alcanzando cifras del $70 \%$ en algunas series, aunque en un 5\% se encuentran implicados más de un organismo, y en el 5-8\% de las ocasiones los hemocultivos son negativos (1-4,6,9,10,13-25).

Por otra parte, la frecuencia de la EI en los pacientes VIH ha disminuido desde la implantación de la terapia antirretroviral (TAR), siendo la mayoría de los casos pacientes que no reciben este tratamiento, y su etiología habitualmente secundaria a bacterias asociadas a la infeccion por HIV (salmonella, streptococo...) $(5,12,23,26)$. Sólo en el caso de la bacteriemia relacionada con catéter, o en las infecciones de piel y tejidos blandos, el agente más frecuente es también el $S$. aureus. Pero si se asocian ambos factores, VIH y ADVP, entonces más del $70 \%$ de las EI es causada por este gérmen $(6,25)$.

La EI de afectación izquierda es menos favorable que la afectación derecha, con peor pronóstico y mayor mortalidad, 
que se sitúa en torno al 20-30\% (2,4,5,8-10,13,20,22). Esta elevada mortalidad en parte es debida a la ausencia de sospecha diagnóstica, a el curso clínico subagudo de la enfermedad y a la frecuente negatividad de los hemocultivos $(9,10,27)$. De todos modos, la afectación de la válvula aórtica y el desarrollo de distrés respiratorio se asocian a una mayor mortalidad reciban o no tratamiento quirúrgico además del médico, constituyendo un factor predictivo de morbimortalidad en los pacientes con endocarditis y empleo de DVP $(10,25)$. Actualmente existe una controversia en cuanto a la supervivencia de los pacientes ADVP que a su vez son VIH. En general se considera que la mortalidad global en los pacientes ADVP es idéntica se encuentren o no infectados por el virus VIH, y que entre los infectados la mortalidad es significativamente superior en aquellos con severa inmunosupresión (CD4 < 200) o con criterios de SIDA, aunque no todos los autores sugieren lo mismo $(1,2,4,6,8-10)$.

Las indicaciones de tratamiento quirúrgico sobre válvula nativa permanecen válidas en este tipo de pacientes $(1,6)$. Por ello, el desarrollo de insuficiencia cardiaca por disfunción valvular, así como la existencia de infección incontrolable son indicaciones absolutas de tratamiento quirúrgico. Además, algunos autores opinan que la EI del lado izquierdo originada por $S$. aureus es difícil de controlar, altamente destructiva y conlleva mortalidad elevada, por lo que debe ser intervenida cuando la respuesta a antimicrobianos no es inmediata y completa. Se considera que la cirugía valvular en los pacientes VIH, independientemente de la utilización de DVP, no comporta peor pronóstico $(1,2,4,10,16)$. Se estima que el trata- miento quirúrgico reduce la mortalidad de la EI izquierda en un $5-10 \%$ de los pacientes, y que la supervivencia en las personas con ambas patologías es del $65 \%$ al año de intervención y del $35 \%$ a los 5-10 años si se combinan tratamiento médico y quirúrgico $(2,7)$, siendo inferior en los pacientes severamente inmunodeprimidos (CD4 < 200) o con criterios de SIDA $(2,4,10,18)$. Tampoco todos los autores comparten esta opinión y consideran que los resultados son peores si el VIH se asocia a la ADVP debido a la alta tasa de recurrencias durante el seguimiento $(16,28)$.

El paciente del presente caso era VIH y ADVP, se encontraba bajo una importante inmunodepresión con unas cifras de CD4 inferiores a 200, hechos que favorecieron el desarrollo de EI.

Poseía una afectación de la válvula aórtica y pulmonar, ambas infrecuentemente afectadas en las diferentes series descritas, tanto de modo aislado como su afectación conjunta. La EI izquierda cursa de modo más insidioso, (lo que unido a la inicial negatividad en los hemocultivos resulta más difícil su diagnóstico) y con peor evolución, con cifras de mortalidad más elevadas, lo que junto con el pobre cumplimiento previo de TAR y la marcada inmunodepresión que presentaba, favorecieron su fallecimiento. Además el paciente presentó en su evolución factores predictivos de mayor mortalidad como son la afectación de la válvula aórtica, y el desarrollo de SDRA. Es posible que el tratamiento quirúrgico se hubiere encontrado indicado en un primer momento, si bien no existe un acuerdo entre todos los autores, ya que algunos sugieren que el avanzado grado de inmunodepresión como en el caso expuesto se asocia a una desfavorable evolución posquirúrgica.

\section{Bibliografía}

1. Miro JM, Moreno A, Mestres CA. Infective Endocarditis in Intravenous Drug Abusers. Curr Infect Dis Rep 2003; 5: 307-16.

2. Miro JM del Rio A Mestres CA. Infective endocarditis and cardiac surgery in intravenous drug abusers and HIV-1 infected patients. Cardiol Clin 2003; 21: 167-84, v-vi.

3. Corti ME, Palmieri OJ, Villafane MF, Trione N. Evaluación de 61 episodios de endocardtis infecciosa en adictos a drogas via parenteral e infeccion por el virus de la inmunodeficiencia humana tipo 1 . Rev Argent Microbiol 2004; 36: 85-7.

4. Miro JM, del Rio A, Mestres CA. Infective endocarditis in intravenous drug abusers and HIV-1 infected patients. Infect Dis Clin North Am 2002; 16: 273-95, vii-viii.

5. Valencia Ortega ME, Guinea J, Ennrique A, et al. Study of 42 cases of infective endocarditis in the HAART era in Spain. Clin Microbiol Infect 2003; 9: 1073-5.

6. Losa JE, Miro JM, Del Río A, Moreno-Camacho A, García F, Claramonte X, et al. Hospital Clinic Endocarditis Study Group. Infective endocarditis not related to intravenous drug abuse in HIV-1-infected patients: report of eight cases and review of the literature. Clin Microbiol Infect 2003; 9: 45-54.

7. Currie PF, Sutherland GR, Jacob AJ, Bell JE, Brettle RP, Boon NA. A review of endocarditis in acquired immunodeficiency syndrome and human immunodeficiency virus infection. Eur Heart J 1995; 16 (Supl. B): $15-8$.

8. Ribera E, Miro JM, Cortes E, Cruceta A, Merce J, Marco F, Planes A, Pare JC, Moreno A, Ocana I, Gatell JM, Pahissa A. Influence of human immunodeficiency virus 1 infection and degree of immunosuppression in the clinical characteristics and outcome of infective endocarditis in intravenous drug users. Arch Intern Med 1998; 158: 2043-50.
9. González Lahoz J, Valencia Ortega ME, Guinea Esquerdo J, Enríquez Crego A, Moreno Celda V, Laguna Cuesta F, et al. Endocarditis de válvulas izquierdas en pacientes con infección por el VIH. An Med Interna (Madrid) 1999; 16: 171-4.

10. Cicalini S, Forcina G, De Rosa FG. Infective endocarditis in patients with human immunodeficiency virus infection. J Infect 2001; 42: 26771.

11. Gebo KA, Burkey MD, Lucas GM, Moore RD, Wilson LE. Incidence of, risk factors for, clinical presentation, and 1-year outcomes of infective endocarditis in an urban HIV cohort. J Acquir Immune Defic Syndr 2006; 43: 426-32.

12. Kohli R, Lo Y, Howard AA, Buono D, Floris-Moore M, Klein RS, et al. Mortality in an urban cohort of HIV-infected and at-risk drug users in the era of highly active antiretroviral therapy. Clin Infect Dis 2005; 41: 864-72.

13. Espinosa Parra FJ, Ramos Rincón JM, Alemán Lorenzo A, Pretel Serrano L, Herrero Huerta F. Infective endocarditis in intravenous drug users. Study of 34 cases. An Med Interna (Madrid) 2000; 17: 356-60.

14. Faber M, Frimodt-Moller N, Espersen F, Skinhoj P, Rosdahl V. Staphylococcus aureus endocarditis in Danish intravenous drug users: High proportion of left-sided endocarditis. Scand J Infect Dis 1995; 27: 4837.

15. Seghatol F, Grinberg I Left-sided endocarditis in intravenous drug users: A case report and review of the literature. Echocardiography 2002; 19: 509-11

16. Carozza A, De Santo LS, Romano G, Della Corte A, Ursomando F, Scardone M, et al. Infective endocarditis in intravenous drug abusers: patterns of presentation and long-term outcomes of surgical treatment. J Heart Valve Dis 2006; 15: 125-31. 
17. Chao TH, Li YH, Tsai WC, Lin LJ, Chen JH, Tsai LM, et al. Clinical characteristics and prognostic determinants of infective endocarditis in adult intravenous drug users. J Formos Med Assoc 2004; 103: 75460.

18. Mestres CA, Chuquiure JE, Claramonte X, Muñoz J, Benito N, Castro MA, et al. Long-term results after cardiac surgery in patients infected with the human immunodeficiency virus type-1 (HIV-1). Eur J Cardiothorac Surg 2003; 23: 1007-16.

19. Rodríguez E, Nonell F, Poblet T, et al. Endocarditis aórtica por Salmonella sp. en una mujer infectada por el virus de la inmunodeficiencia humana. An Med Interna (Madrid) 2002; 19: 60-1.

20. Aguilar JA. Summerson C. Infective endocarditis in intravenous drug addicts. Arch Inst Cardiol Mex 2000; 70: 384-90.

21. Rivera Del Río JR, Flores R, Meléndez J, Gómez MA, Vila S, Hunter R. Profile of HIV patients with and without bacterial endocarditis. Cell Mol Biol 1997; 43: 1153-60.

22. Ulanova VI, Tsinzerling VA. Clinical and morphological characteristics of infective endocarditis in HIV-infected drug addicts. Arkh Patol 2006; 68: 14-8

23. Gebo KA, Burkey MD, Lucas GM, Moore RD, Wilson LE. Incidence of, risk factors for, clinical presentation, and 1-year outcomes of infective endocarditis in an urban HIV cohort. J Acquir Immune Defic Syndr 2006; 43: 426-32

24. de Alarcon A, Villanueva JL. Endocarditis in parenteral drug addicts. Right-sided endocarditis. Influence of HIV infection. Rev Esp Cardiol 1998; 51 (Supl. 2): 71-8.

25. Mathew J, Addai T, Anand A, Morrobel A, Maheshwari P, Freels S. Clinical features, site of involvement, bacteriologic findings, and outcome of infective endocarditis in intravenous drug users. Arch Intern Med 1995; 155: 1641-8.

26. Sani MU, Okeahialam BN, Aliyu SH, Enoch DA. Human immunodeficiency virus (HIV) related heart disease: A review. Wien Klin Wochenschr 2005; 117: 73-81.

27. Ruiz Junior E, Schirmbeck T, Figueiredo LT. A study of infectious endocarditis in Ribeirao Preto, SP-Brazil. Analysis of cases occurring between 1992 and 1997. Arq Bras Cardiol 2000; 74: 217-31.

28. Chong T, Alejo DE, Greene PS, Redmond JM, Sussman MS, Baumgartner WACameron DE. Cardiac valve replacement in human immunodeficiency virus-infected patients. Ann Thorac Surg 2003; 76 : 478-80. 